\title{
Soft X-ray Emission Spectroscopy of Borosilicate Glasses and Minerals by SXES and EPMA-WDS
}

\author{
Anette von der Handt ${ }^{1 *}$, Jed Mosenfelder ${ }^{1}$ \\ 1. University of Minnesota, Dept of Earth Sciences, Minneapolis, USA \\ * Corresponding author: avdhandt@umn.edu
}

Borosilicate glasses have been extensively investigated for their chemical, electrical and optical properties in material and environmental sciences and are being proposed as potential encapsulation materials for the storage of high-level nuclear waste, because of their high chemical durability. A robust assessment of the long-term stability of these glasses relies on the knowledge of alkali borosilicate glass structure and composition due to the importance of structural controls on physico-chemical properties of glasses.

However, the analysis of glasses and geological materials for boron by EPMA has been historically fraught with many complications: (1) low yields and absorption of X-rays, (2) peak shift and shape alteration, (3) interferences caused by higher order X-ray lines, (4) inadequate matrix correction procedures, and (5) artefacts induced by beam damage and hydrocarbon contamination during analysis [1]. The original WDS lead stearate crystals (STE) used for boron analysis had poor sensitivity but a good enough spectral resolution to facilitate spectroscopic investigations of boron-bearing materials. Their sensitivity for peak shape alterations in turn was problematic for quantitative analysis and consequently they have been almost exclusively replaced on modern microprobes by the newer layered synthetic microstructure (LSM) crystals. These provide increased peak intensities (up to 50x depending on type and analytical conditions), but substantially broaden the boron peak relative to STE crystals. The boron peak at full-width half-maximum (FWHM) is approximately twice as wide using the $\mathrm{Mo} / \mathrm{B}_{4} \mathrm{C}$ LSM (LDEB, $14.5 \mathrm{~nm}$ ) compared with the same peak measured using STE [2], limiting its use for spectroscopic analysis. In addition, problems with fluorescence of the $\mathrm{B}$ atoms in the $\mathrm{B}_{4} \mathrm{C}$ layer [3] and strong refraction effects leading to spectral shifts [4] further complicate the use of LSM crystals for spectroscopic applications.

In recent years, new tools have become available for light element analysis including a new generation of LSM (LDE6, Cr/C, 12nm) as well as a soft X-ray emission spectrometer (SXES, JEOL, Inc.). The SXES is composed of a CCD area detector that can be coupled to multiple varied-line-spacing (VLS) aberration-corrected gratings (JS50XL, JS200N, JS300N and JS2000) that cover a combined energy range from $50 \mathrm{eV}$ to $2.3 \mathrm{keV}$. It provides higher spectral resolution relative to traditional wavelength dispersive spectrometers (WDS), yielding information on electronic structure and bonding.

Using our JEOL JXA-8530FPlus electron microprobe, equipped with both SXES and a LDE6L monochromator, we investigated a range of borosilicate materials in order to explore the potential of these detectors for spectroscopic and quantitative analysis. Analyses were carried out at different accelerating voltages $(2,5,7$, and $10 \mathrm{kV})$ on four borosilicate glasses from NIST as well as a range of borosilicate minerals (axinite, dumortierite, tourmaline, werdingite, grandidierite, datolite, and danburite) with known trigonal and tetrahedral boron coordination.

The SXES - in addition to its higher spectral resolution - provides parallel acquisition over a wider range of the X-ray spectrum. In the case of the JS300N grating that we used, this also allows detection of the second and third order oxygen Ka lines that can carry spectroscopic information. 
We found that, indeed, the FWHM of B Ka on the SXES compares favourably relative to the LDE crystals (Figure 1a) and features in the symmetry and fine structure of boron spectra in our samples is more apparent. Figure $1 \mathrm{~b}$ depicts the large changes observed when going from elemental boron to boron nitride and boron oxide. Satellite bands A and C, present to varying degrees in all our materials with B$\mathrm{O}$ bonds while $\mathrm{C}$ is also present in $\mathrm{BN}$, appear and vary in position and intensity relative to the main boron peak. In combination with peak symmetry changes for $\mathrm{B}$ and $\mathrm{O}$, the SXES brings back the potential for EPMA-based spectroscopy of borosilicate glasses and minerals and other boron-bearing materials.

\section{References:}

[1] GF Bastin, \& HJM Heijligers (in "Electron probe quantitation” (Springer, Boston) p. 145.

[2] J McGee, L Anovitz (1996) in "Boron - Mineralogy, Petrology, Geochemistry, MSA, p. 771.

[3] H Kobayashi, K Toda, H Kohno, T Arai, R Wilson, Advances in X-ray Anal 38 (1995), p. 307.

[4] S Luck, D Urch, D Zhen, X-Ray Spectrometry 21 (1992), p.77.

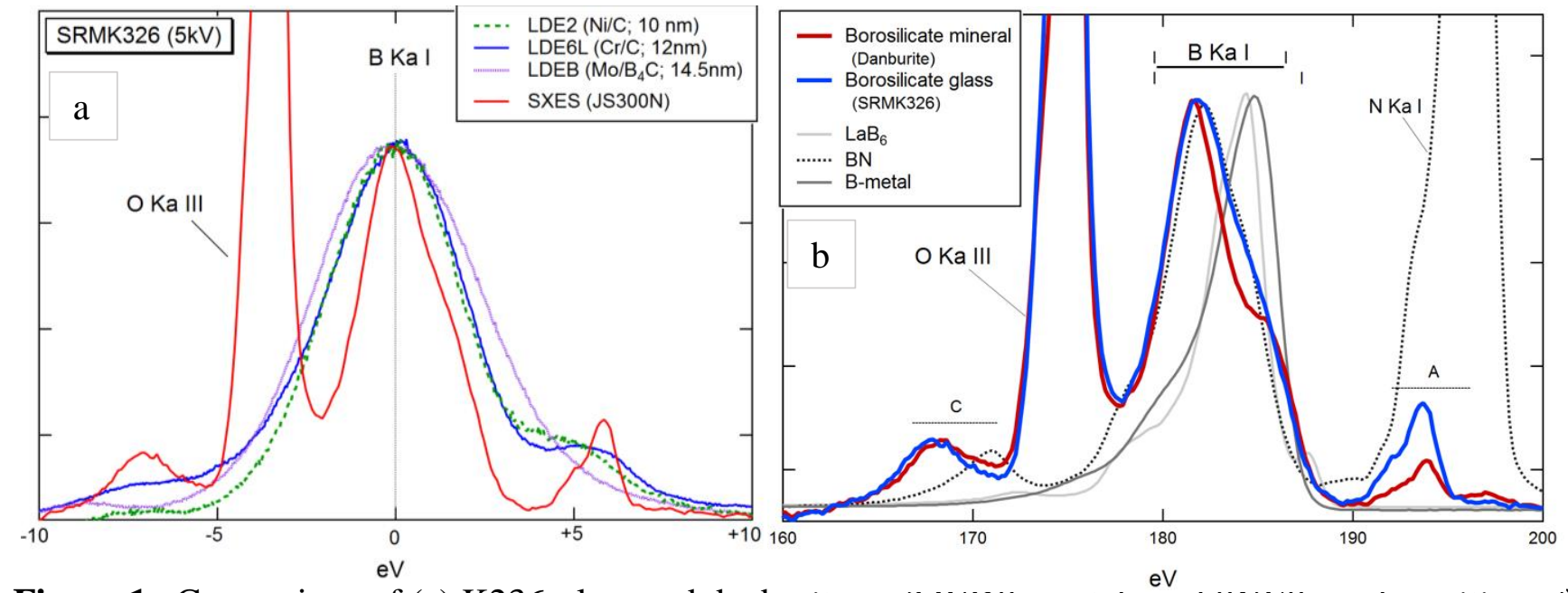

Figure 1. Comparison of (a) K236-glass and danburite on 3 WDS crystals and SXES, peak position of $\mathrm{B} \mathrm{Ka}$ is chosen as the common center, and the spectra are normalized to peak height. (b) B Ka peak shapes and position between different boron-containing materials on the SXES. Intensities are scaled.
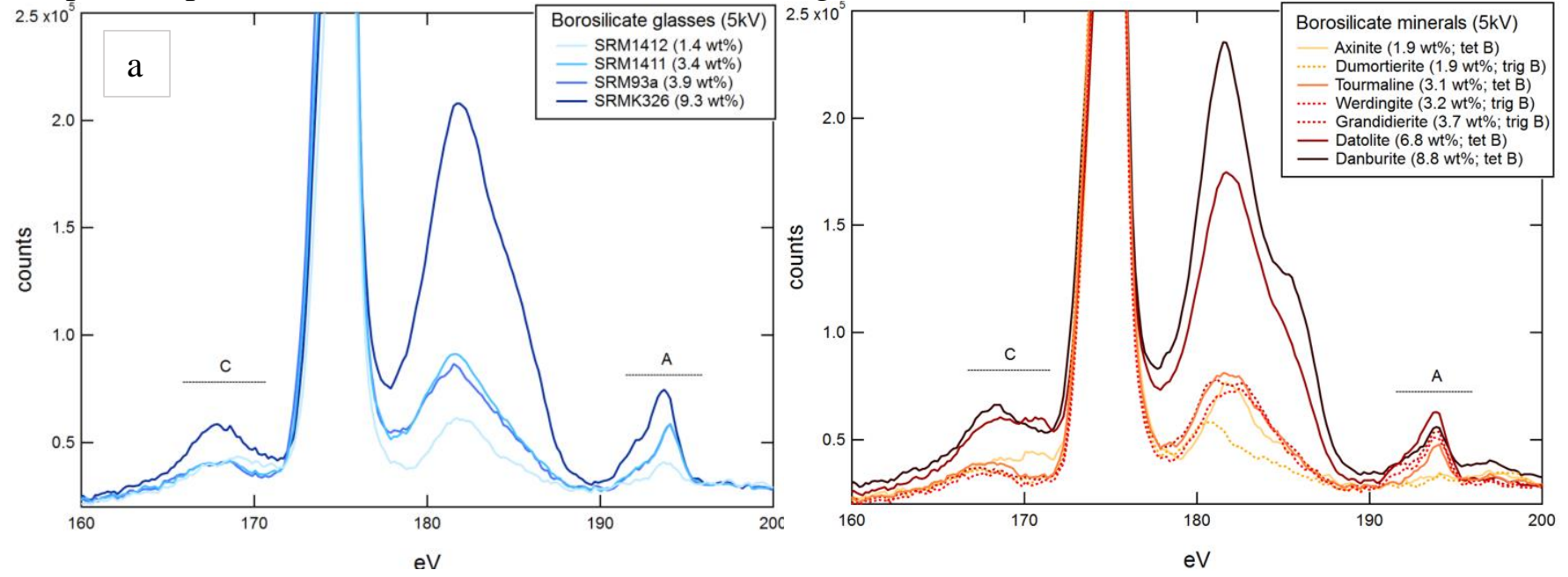

Figure 2. Comparison of (a) B spectra between four borosilicate glass and (b) seven borosilicate minerals with tetragonal or trigonal boron coordination. Note the position and heights of $\mathrm{A}$ and $\mathrm{C}$ satellite peaks. 